\title{
UNA LECTURA SEMIÓTICA DEL COURS EN EL AÑO DE SU CENTENARIO
}

\author{
A SEMIOTIC READING OF THE COURS IN \\ THE YEAR OF ITS CENTENNIAL
}

\begin{abstract}
Mirko LAMPIS
Universidad Constantino el Filósofo de Nitra (República Eslovaca)

mlampis@ukf.sk
\end{abstract}

Resumen: El objetivo de este artículo es el de estudiar y describir los fundamentos teóricos del Cours de linguistique générale, de F. de Saussure ( $1^{\mathrm{a}}$ ed.: 1916). Más concretamente, estudiaremos aquí la concepción de la ciencia que subyace al trabajo de Saussure y su tratamiento de la noción de semiología.

Palabras clave: Semiótica. Ferdinand de Saussure. Cours de linguistique générale.

\begin{abstract}
The goal of this paper is to study and describe the theoretic foundations of the Cours de linguistique générale, by F. de Saussure (1st ed.: 1916). More specifically, we will study here the conception of science that underlies Saussure's work and also his treatment of the notion of semiology.
\end{abstract}

Key Words: Semiotics. Ferdinand de Saussure. Cours de linguistique générale. 


\section{INTRODUCCIÓN}

Existen, antes de la "revolución" física y epistemológica del siglo $\mathrm{XX}$, quizás sólo dos libros que han logrado sacudir y modificar profunda y radicalmente el panorama científico internacional. Los dos, por supuesto, no surgieron de la nada, ya que ningún texto lo hace, pero su rápida difusión y sus duraderos efectos, así como las duras críticas que se les movieron y las incomprensiones -no siempre estériles- que generaron, nos dan la medida de cómo consiguieron condensar, por decirlo así, una serie de problemas y de soluciones cuya urgencia era de algún modo patente en la episteme de su tiempo: Philosophiae naturalis principia mathematica, de Isaac Newton ( $1^{\mathrm{a}}$ ed.:1687), y On the Origins of Species, de Charles Darwin ( $1^{\text {a }}$ ed.:1859); a estas dos obras, sin embargo, personalmente no dudaría en añadir una tercera: el Cours de linguistique générale, de Ferdinand de Saussure ( $1^{\mathrm{a}}$ ed.: 1916).

Ahora que se han cumplido cien años de la primera publicación del Curso, quisiera dedicar unas cuantas páginas a algunos de los problemas tratados en (y abiertos por) este texto fundacional, pero no en la perspectiva de la lingüística o de la historia de la lingüística, sino en la de la semiótica. Son imprescindibles, por lo tanto, según creo, unas cuantas aclaraciones preliminares.

1) Los grandes textos científicos, al igual que las obras de arte, acumulan, sedimentan y abren interpretaciones, debido esto, esencialmente, a su gran densidad semiósica, a las ambigüedades y contradicciones que con frecuencia a esta densidad acompañan y al hecho de que sus isotopías de sentido se pueden organizar e interrelacionar de diferentes maneras, definiéndose inevitablemente mucho más allá de los límites y subdivisiones intratextuales.

Aquí, más que al "texto Saussure", nos dedicaremos a ese concreto "objeto textual" que es el libro titulado Cours de linguistique générale, tal y como salió, por así decirlo, de la pluma de sus editores en $1916-\mathrm{y}$ luego, con ligeras correcciones, en 1922- y tal y como ha llegado, edición tras edición, ejemplar tras ejemplar, hasta hoy en día. Más concretamente, examinaremos una de las numerosas traducciones de este "objeto textual": su primera traducción al español, que debemos a Amado Alonso, puesto que representa la vulgata que más ha influido en el ámbito del mundo 
hispánico, al que me dirijo ${ }^{1}$.

Además del Curso, emplearé también los Escritos sobre lingüística general (Saussure, 2002), obra que reúne unas notas y apuntes manuscritos descubiertos sólo en 1996, más una serie de conferencias y notas que ya había editado R. Engler en 1968 y 1974. Este material es de gran interés ya que permite probar, ahondar y matizar, cuando es preciso, la terminología y las teorías saussureanas así como se exponen (y "vulgarizan") en el Curso.

2) No pretendo en esta sede -ni de todas formas podría- armar una reconstrucción o refundación "filológica" del pensamiento y de las teorías de Saussure, en búsqueda de una auténtica y genuina, aunque hipotética, intentio autoris. Así como sostiene De Mauro (1967, 1967b), la labor de los editores del Curso, Bally y Sechehaye, modificó de manera sustancial el pensamiento del maestro suizo, empezando por la radical reordenación de los temas tratados en los tres cursos de lingüística general que Saussure impartió en Ginebra entre 1906 y 1911, pasando por elecciones compositivas y terminológicas de tipo uniformador, hasta llegar a añadidos y "empalmes" textuales que también, más allá de la necesidad de dar coherencia y cohesión a un texto construido a partir de materiales fragmentarios y no definitivos, tuvieron el efecto de "fijar" y "orientar" -cuando no tergiversar- unas teorías lingüísticas extremamente complejas y todavía in fieri. A esta labor de reelaboración a posteriori y en ocasiones arbitraria -a la que también se refieren los propios editores en su introducción al Curso, al parecer conscientes de las dificultades y peligros que esta entrañaba- se deben, más que a otra cosa, en opinión de De Mauro, muchas de las críticas que tradicionalmente se le han dirigido a Saussure, empezando por la acusación de defender un planteamiento radicalmente anti-historicista o a-historicista.

Así como no es nuestro objetivo reconstruir la "genuina" teoría saussureana, tampoco lo es ahondar en las críticas dirigidas a Saussure y propiciadas por una serie de incomprensiones (y aun ignorancias) debidas también a la organización que los editores dieron al Curso (aunque sí dedicaremos observaciones puntuales a determinados aspectos del problema). Sin embargo, quisiera precisar que, en mi opinión, si De Mauro está en lo cierto en subrayar que la dimensión histórica y social

1. Se trata, por lo demás, de una traducción que el propio Tullio De Mauro (1967b: 342) definió como "excelente". 
de la lengua -desde un punto de vista ontológico, cabría añadir- es una directa y necesaria consecuencia del principio de la arbitrariedad del signo lingüístico, no por ello se vuelve menos patente, en Saussure, la necesidad metodológica de deshistoricizar el estudio de la lengua o, para decirlo mejor, de fundamentar todo estudio lingüístico a partir de la individuación de una estructura acrónica de oposiciones relevantes.

Volveremos sobre este punto en la segunda parte del texto, pero ya ahora quiero señalar que son las reservas y las soluciones metodológicas de Saussure, motivadas por la necesidad de dar una fundamentación racional y rigurosa a la disciplina lingüística, lo que influirá profundamente en la elaboración de la semiótica contemporánea, y que tales reservas y soluciones, si bien no transforman a Saussure en ese acérrimo enemigo del estudio de la parole y de las dinámicas diacrónicas que a veces parece asomarse desde las páginas y entre las líneas de sus críticos -y también de algunos pasajes del Curso, a decir verdad-, sí acercan la teoría saussureana a posiciones que podríamos definir, con palabras de González de Ávila (2002), como aislantes e inmanentistas.

3) Aunque en el Curso, si no yerro, la palabra "estructura" se emplea sólo tres veces, y siempre en un sentido no particularmente técnico (Saussure, 1945 [1916]: 218, 284, 298), lo cierto es que las principales nociones que rigen el enfoque saussureano -las nociones de valor, signo, significante, significado, relación sintagmática y relación asociativa, más las dicotomías ${ }^{2}$ parole-langue y diacronía-sincronía- son la base y el punto de arranque -positivo, y en ocasiones polémico- de la epistemología estructuralista, incluidas sus vertientes funcionalista y generativa (Hjelmslev, 1961; Fabbri y Marrone, 2000).

No creo que haga falta recordar la importancia del estructuralismo no sólo en lingüística, sino también en antropología, psicología, mitología y literaturología; o en semiótica. Pero sí creo que vale la pena reflexionar acerca de los fundamentos, las consecuencias y el modus operandi semióticos del Curso. Sobre todo porque la obra, en tanto que texto fundacional $-\mathrm{y}$ su autor, en tanto que autoridad arqueológica-, sigue dialogando con la semiótica y con sus especialistas, quienes siguen interrogándola, comentándola y discutiéndola.

Al tratar el problema de la enunciación y de ese elemento "espurio"

2. Acerca de la noción de "dicotomía", véase Štúr, 2013. 
que es el enunciador individual ("espurio" porque toda enunciación es un acto de parole, una actualización particular y contingente de la langue), comenta Bertrand que en la semiótica contemporánea se han venido delineando dos grandes tendencias:

por un lado, la de Trubeckoj, de Brøndal, de Jakobson, de Benveniste, inspirada en la filosofia husserleana y a la que se pueden reconducir las tesis formuladas por el propio Coquet. Por otro lado, la de Saussure, de Hjelmslev y de Greimas, de estricta observancia formalista, logicista y estructuralista. La historia, en este caso, se pone al servicio del debate que polémicamente postula la existencia de dos paradigmas contrapuestos: una semiótica de la realidad opuesta a una semiótica de la inmanencia, una semiótica de la enunciación opuesta a una del enunciado, una aproximación subjetivista opuesta a una objetivista, una visión del sentido como continuum a una visión del sentido como entidad discontinua fundada en oposiciones categoriales (Bertrand, 2000: 68; la traducción es mía).

Sin considerar ahora que existe también una tercera tendencia, una semiótica que no reconoce $\mathrm{u}$ otorga un valor teórico fuerte a la distinción entre enunciación y enunciado ${ }^{3}$, creemos que es precisamente esta "observancia formalista, logicista y estructuralista" de la que habla Bertrand la herencia más destacada - o la contribución, si herencia parece término demasiado comprometedor-que la semiótica contemporánea, vía Hjelmslev, Barthes y Greimas, le reconoce a Saussure.

4) Lejos de pretender llevar a cabo un análisis exhaustivo del Curso, mi objetivo es el de definir y emprender la lectura de unos cuantos recorridos textuales específicos a la luz y bajo la guía de unos saberes semióticos dados, subrayando al mismo tiempo los aspectos teóricos y metodológicos del Curso que más han contribuido, en mi opinión, a la definición de esos mismos saberes.

Nos hallamos, evidentemente, ante un círculo: la interpretación

3. Podemos recordar, de paso, la no muy bien declarada tendencia por parte de los autores que se adhieren al paradigma estructuralista a presentar su propia semiótica como la semiótica. 
del Curso como base de la semiótica contemporánea, la semiótica contemporánea como base de la interpretación del Curso. Pero así funcionan las cosas, por decirlo a modo de sentencia, en el mundo textual de la cultura, de modo que no estimo necesario intentar explicar o justificar, y menos aún enmendar, esta circularidad. Los textos nacen de interpretaciones, los textos producen interpretaciones; o también: las interpretaciones generan textos que generan interpretaciones que generan textos... sin que resulte posible, comúnmente, fijar límites demasiado netos o definitivos en el proceso. Y no queremos, con esto, abogar por ningún derecho a la "interpretación salvaje", sino sencillamente recordar que en la complejidad de las relaciones intra-, inter- y extra-textuales se juegan los desplazamientos, los equilibrios y las coherencias que hacen del texto un objeto interpretable.

5) Los recorridos textuales que me propongo examinar son los siguientes:

- primer recorrido: hacia una ciencia del lenguaje;

- segundo recorrido: la semiología;

- tercer recorrido: del habla a la lengua;

- cuarto recorrido: el problema del tiempo;

- quinto recorrido: forma y sustancia, potencia y acto;

- sexto recorrido: el significado.

Puesto que, una vez desarrollados, estos seis temas excederían con creces los límites de espacio que se suelen asignar a un artículo científico, no me queda más remedio que dividirlos. Voy a presentar en esta primera parte de mi texto, pues, sólo los dos primeros recorridos, dejando los restantes cuatro para una segunda y una tercera parte que se publicarán (si hay suerte) en otro momento.

\section{PRIMER RECORRIDO: HACIA UNA CIENCIA DEL}

\section{LENGUAJE}

El punto de partida y, a la vez, el "eje" o "pivote" alrededor del cual girará y oscilará nuestra lectura del Curso es la siguiente constatación: la teoría saussureana nace y toma forma a partir de la exigencia de dar un estatus científico riguroso a la lingüística. Sobre todo porque, como señaló el propio Saussure (1945 [1916]: 48), aunque "en la vida de los individuos y en la de las sociedades no hay factor tan importante como 
el lenguaje", lo cierto es que, al considerar su estudio, cabe concluir que "no hay terreno donde hayan germinado más ideas absurdas, prejuicios, espejismos y ficciones". No parece, pues, que Saussure estuviera muy conforme con el estado de los estudios lingüísticos de su tiempo, dato que subrayan también los editores del Curso al empezar su introducción:

Muy a menudo oímos a Ferdinand de Saussure deplorar la insuficiencia de los principios y de los métodos que caracterizaban a la lingüística en cuyo ambiente había crecido su genio, y toda la vida buscó obstinadamente las leyes directrices que pudieran orientar su pensamiento a través de ese caos (Bally y Sechehaye, en Saussure, 1945 [1916]: 31).

También Ducrot y Todorov, al introducir la entrada Saussurianismo de su Diccionario enciclopédico de las ciencias del lenguaje, se mueven en la misma dirección:

Después de escribir, a los veintiún años, una Memoria sobre el sistema primitivo de las vocales indoeuropeas (París, 1878), obra que se destaca entre los aciertos de la escuela neogramática, el lingüista suizo Ferdinand de Saussure abandona casi por completo las investigaciones lingüísticas porque sus fundamentos le parecen inciertos y piensa que tales estudios deben postergarse hasta que se revisen por completo las premisas de la lingüística. Él mismo intenta esa revisión y presenta los resultados de sus trabajos en tres cursos dados en Ginebra entre 1906 y 1911, y publicados tres años después de su muerte por algunos de sus discípulos, con el título de Curso de lingüística general (París, 1916) (Ducrot y Todorov, 1972: 29).

Hay que reconocer, sin embargo, que si la lingüística se encontraba, según Saussure, en un estado caótico, no era sólo por la insuficiencia de sus principios y métodos disciplinares: su propio objeto de estudio, el lenguaje humano, es una materia extraordinariamente compleja que difícilmente se presta a algún tipo de sistematización racional. 
Tomado en su conjunto, el lenguaje es multiforme $y$ heteróclito; a caballo en diferentes dominios, a la vez fisico, fisiológico y psíquico, pertenece además al dominio individual y al dominio social; no se deja clasificar en ninguna de las categorías de los hechos humanos, porque no se sabe cómo desembrollar su unidad (Saussure, 1945 [1916]: 51).

Dicho "caos" sólo podía ser afrontado y superado con espíritu y, diremos, con voluntad de científicos. Sin querer otorgar -o restar- excesiva importancia a un dato meramente biográfico, no se olvide que Saussure creció rodeado, en su ambiente familiar, de científicos y de recuerdos de científicos, naturalistas, químicos, físicos y geólogos (De Mauro, 1967b: 286-287), y que por ende bien conocía cuáles eran los objetivos y los requisitos de la "ciencia" tal y como se practicaba en su tiempo ${ }^{4}$. Léanse, sin más, los objetivos que Saussure asigna a la lingüística:

a) hacer la descripción y la historia de todas las lenguas de que pueda ocuparse [...];

b) buscar las fuerzas que intervengan de manera permanente y universal en todas las lenguas, y sacar las leyes generales a que se puedan reducir todos los fenómenos particulares de la historia;

c) deslindarse y definirse ella misma

(Saussure, 1945 [1916]: 46).

Reconocemos, en el punto 'a', una admisión del valor general de los estudios lingüísticos; y reconocemos, en los puntos 'b' y 'c', los objetivos mismos del Curso.

El punto 'c', en particular, reviste una importancia fundamental para Saussure, ya que sin esa "operación elemental" que es "determinar la naturaleza de su objeto de estudio", "una ciencia es incapaz de procurarse un método" (Saussure, 1945 [1916]: 42). Como también comenta Coquet:

4. Desde esta perspectiva, diría que cobran nuevo interés, en el Curso, las frecuentes comparaciones entre la lingüística y las demás ciencias, por un lado, y los numerosos símiles inspirados en el mundo de las ciencias naturales, por otro. 
Afirmar la autonomía de sus disciplinas: este era, a comienzos del siglo $X X$, el proyecto cientifico común a sociólogos y lingüistas. La lengua, así como el "pensamiento colectivo", debe ser estudiada en sí misma

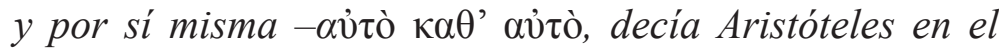
Organon-conservando la especificidad de cada término de la proposición (Coquet, 2008: 62; la traducción es mía).

Así pues, si ya Benveniste (1975: 87-88) subrayó que para Saussure el proyecto de la lingüística sólo era posible delimitando su objeto de estudio y descubriendo el principio de unidad que domina la multiplicidad de las manifestaciones del lenguaje, hay que añadir que tales requisitos se inspiraban y enmarcaban en una concepción general, y por entonces canónica, del correcto quehacer científico.

A fin de afirmar y establecer la autonomía de la ciencia de la lengua, Saussure llevó adelante una doble estrategia legitimadora, operando tanto en la vertiente externa, o inter-disciplinaria, de la lingüística como en su vertiente interna, o intra-disciplinaria. Por el lado externo, la consigna era la de establecer conexiones claras entre la lingüística y las demás disciplinas científicas; es decir: buscar y definir el lugar que le corresponde a la lingüística en el sistema de las ciencias. Por el lado interno, se trataba de delinear y describir de la forma más precisa y completa posible su objeto de estudio y su metodología. A la primera consigna podemos reconducir la noción saussureana de semiología; a la segunda, la noción de lengua (dedicaremos a tales nociones, respectivamente, nuestro segundo y tercer recorrido).

Es, en suma, la exigencia de una racionalización rigurosa del estudio del lenguaje humano - estudio hasta entonces "caótico"- lo que motiva las principales elecciones teóricas y metodológicas de Saussure y, asimismo, cabe suponer, las principales elecciones textuales de sus editores. Hasta llegar a la célebre sentencia con la que se cierra el Curso: "la lingüística tiene por único y verdadero objeto la lengua considerada en sí misma, y por sí misma" (Saussure, 1945 [1916]: 364).

Tal como apunta De Mauro (1967b: 455), es bien posible que esta frase sea una libre creación -o manipulación- de los editores, ya que no hay constancia de ella en los apuntes que se han conservado de Saussure 
y de sus alumnos. Los editores, probablemente, pensaron "resumir" con esta fórmula el "hallazgo" más importante de Saussure, el eje de todo su sistema teórico, y lo cierto es que muchos continuadores del lingüista suizo se movieron en la misma dirección:

buena parte de la lingüistica de inspiración estructuralista ha creído que respetar a $S$. significaba hacer caso omiso de los desequilibrios del sistema, de la dinámica sincrónica, de los condicionamientos sociales, de los fenómenos evolutivos, del nexo entre estos y las diferentes contingencias históricas, de todo el fluctuar de fenómenos lingüisticos de los y por los que la lengua es forma (De Mauro, 1967b: 456; la traducción es mía).

Según De Mauro, en cambio, jamás sostuvo Saussure que el único y verdadero "objeto de estudio" de la lingüística es la langue, el sistema acrónico de las oposiciones relevantes, siendo este sistema, más bien, "norma y forma de una materia extremadamente heterogénea y variada que toda cabe en el legítimo dominio del estudio lingüístico"; el sistema, en otros términos, sería para Saussure el "objet específico de la lingüística" sólo en la medida en que constituye el "principio ordenador de los conocimientos lingüísticos" (ibíd.).

$\mathrm{Al}$ respecto, quisiera añadir que, si no separamos convenientemente la ontología de la lengua de la metodología de su estudio (y aun de las condiciones de su conocimiento), corremos el riesgo de tergiversar, una vez más, el pensamiento saussureano. Así pues, si tiene razón De Mauro en sostener que la arbitrariedad del signo lingüístico es directa consecuencia y garantía última de la historicidad de la lengua, fenómeno necesariamente social y contingente, también es cierto que esa misma arbitrariedad conduce a la necesidad de considerar la lengua como un hecho esencialmente abstracto y sincrónico (so pena de volver al "caos" de los estudios lingüísticos pre-saussureanos): "como el signo lingüístico es arbitrario por naturaleza, parece a primera vista que nada impide [ ] un sistema libre que sólo dependa de sus principios lógicos, y como una ciencia pura de relaciones abstractas" (Saussure, 2002: 293).

En definitiva, la estrategia científica que Saussure quiere llevar a cabo es, desde el punto de vista metodológico, aislante y reduccionista: 
enfrentada a una realidad "caótica" de hechos y fenómenos, tarea de la ciencia es la de simplificar dicha realidad, abstrayendo y aislando (y casi se podría decir: "descorporeizando") un sistema limitado y estable de leyes, relaciones y elementos y reduciendo a su funcionamiento la pluralidad de las manifestaciones observadas. La lingüística debe, en otros términos, buscar y aislar patrones formales regulares, o "tipos generales", en palabras del propio Saussure, "tipos generales" que sólo resultan posibles "por el recuerdo de un número suficiente" de formas similares y que sólo "tienen su base en la lengua en forma de recuerdos concretos" (Saussure, 1945 [1916]: 210).

Se trataba de acatar, en suma, los dos principios que han guiado el correcto quehacer de la ciencia occidental desde el siglo XVII hasta el siglo XX (y de los que Newton y Darwin son, precisamente, dos preclaros exponentes):

i) hay que reducir lo complejo a lo simple;

ii) hay que reducir la pluralidad a la unidad.

Principios perfectamente aplicables en lingüística, siempre y cuando nos centremos en lo esencial: el sistema regular de la lengua, tal y como se define en el uso social (con sus hábitos y convenciones transindividuales) y tal y como se graba y reproduce en la mente (en el cerebro) de todos los hablantes.

Como también sostiene Saussure en el Curso (1945 [1916]: 190), si la lingüística pudiera determinar los elementos que maneja, "cumpliría su tarea completa, pues habría reducido todos los fenómenos de su competencia a su principio primordial"; o en uno de sus apuntes: "El mecanismo de la lengua -contemplada en su totalidad EN UN MOMENTO DADO, que es el único modo de estudiar su mecanismo- un día quedará reducido a fórmulas relativamente sencillas" (Saussure, 2002: 47). Objetivo irrealizado y difícil de realizar, pero necesario e inaplazable si la lingüística quiere cumplir con su cometido de ciencia rigurosa.

\section{SEGUNDO RECORRIDO: LA SEMIOLOGÍA}

Si mi recuento no yerra, el sustantivo "semiología" y el adjetivo "semiológico" en el Curso aparecen, respectivamente, trece ${ }^{5}$ y diez veces.

5. Once, en realidad, si descontamos un título y una nota a pie de página, ocurrencias que con toda 
Pudiera parecer poco, pero habría que considerar también que el Curso no es un libro que verse sobre semiótica, sino sobre lingüística, y que la semiótica, hecho aún más importante, a comienzos del siglo XX ni siquiera existía (aunque Peirce, apasionado inventor de nuevas disciplinas, al otro lado del Atlántico y sin que Saussure tuviera noticia de ello, en aquellos mismos años estaba elaborando su propia teoría semiótica).

En efecto, Saussure llegó a convencerse de la conveniencia -y aun de la necesidad- de una "disciplina general de los signos" - de la que la lingüística, a la postre, no sería más que una rama especializada-bien antes de impartir sus cursos de lingüística general, como demuestra el hecho de que el profesor Adrien Naville, de la Facultad de Letras y Ciencias Sociales de la Universidad de Ginebra, incluyera la semiología saussureana en la edición de 1901 de su obra Classification des Sciences (1 ${ }^{\mathrm{a}}$ ed.: 1888). La "presentación oficial" de la semiología, por lo tanto, es anterior de quince años a la publicación del Curso. Escribe Naville:

M. Ferdinand de Saussure insiste en la importancia de una ciencia muy general, que él llama semiología y cuyo objeto serían las leyes de la creación y transformación de los signos y de su significado ${ }^{6}$. La semiología es una parte esencial de la sociología. Como el más importante de los sistemas de signos es el lenguaje convencional de los hombres, la ciencia semiológica más avanzada es la lingüística o ciencia de las leyes de la vida del lenguaje. La fonología y la morfología tratan sobre todo de las palabras, la semántica del significado de las palabras; querer separar estos estudios el uno del otro equivaldría a mal comprender sus objetos. Los lingüistas actuales han renunciado a las explicaciones puramente biológicas (fisiológicas) en fonología y consideran con razón la lingüistica entera como una ciencia psicológica.

La lingüística es, o al menos tiende a ser cada vez más,

evidencia debemos a los editores; además, seis ocurrencias se concentran sólo en el tercer apartado del tercer capítulo de la Introducción, dedicado, precisamente, al "Lugar de la lengua en los hechos humanos: la semiología”.

6. Recordemos que Naville subdivide todas las ciencias en tres grupos: las ciencias de las leyes, o teoremática, las ciencias de los hechos, o historia, y las ciencias de las reglas ideales de acción, o canónica. 
una ciencia de las leyes; ella se distinguirá cada vez más netamente de la historia de la lengua y de la gramática (Naville, 1901: 104; la traducción es mía).

También en las tablas finales que resumen su clasificación de las ciencias, Naville incluye la "lingüística" entre las ciencias nomotéticas y, más concretamente, entre las ciencias psicológicas, mientras que rubrica la "historia lingüística" entre las ciencias históricas; como decir: si la lingüística sincrónica descubre leyes, la lingüística diacrónica describe hechos; sus objetos, así como sus objetivos, son harto diferentes.

En una de las notas de Saussure, posterior a la edición de 1901 del libro de Naville -convenientemente citado en el texto-, encontramos el siguiente apunte:

Ha habido discusiones para saber si la lingüistica pertenecía al orden de las ciencias naturales o al de las ciencias históricas. No pertenece a ninguno de los dos, sino a un compartimiento de las ciencias que, si no existe, debería existir con el nombre de semiología, es decir, ciencia de los signos o estudio de lo que se produce cuando el hombre trata de significar su pensamiento por medio de una convención necesaria. Entre todos los sistemas semiológicos el sistema "lengua" es el único [...] que ha tenido que enfrentarse a la prueba de encontrarse en presencia del Tiempo, que no se basa simplemente en el mutuo consentimiento de vecino a vecino, sino también en la relación de padre a hijo por imperiosa tradición y está sometido al azar de lo que ocurra en esta tradición [...]. Este hecho [...] es ignorado por los filósofos: ninguno de ellos enseña lo que sucede en la transmisión de una semiología. $Y$ en cambio ese mismo hecho acapara de tal modo la atención de los lingüistas que estos llegan a creer que su ciencia es histórica o eminentemente histórica, cuando no es nada más que semiológica; por eso está enteramente incluida en la psicología, con tal de que esta por su parte comprenda que tiene en la lengua un objeto que se extiende a través del tiempo y la obliga absolutamente a salir de 
sus especulaciones sobre el signo momentáneo y la idea momentánea (Saussure, 2002: 234-235).

Si la lingüística es una ciencia, pues, no es una ciencia natural (o fisiológica), y tampoco histórica, sino semiológica. El hecho de que la lengua esté expuesta a los azares de la tradición y a los efectos del tiempo produce ese "desdoblamiento necesario" de la lingüística en sincrónica y diacrónica, pero no modifica lo esencial de su objeto específico, el cual, más allá de sus concretas realizaciones $-\mathrm{O}$ actualizaciones-contingentes, fisiológicas e históricas, es y sigue siendo un sistema de oposiciones relevantes en la conciencia de los miembros de una colectividad hablante.

Por ello, también llama la atención, en la cita anterior, el sintagma "transmisión de una semiología", pues parece que aquí el término "semiología" no remite tanto a un tipo de estudio como a un objeto de estudio; un objeto de estudio que, en otro interesante apunte saussureano, es definido explícitamente como "un sistema de signos totalmente independiente de sus antecedentes y tal como existe en la mente de los sujetos hablantes" (Saussure, 2002: 47). Se da, también en este caso, cierta oscilación terminológica por parte de Saussure, luego convenientemente "depurada" en el Curso?.

Ahora bien, tal y como se desprende de los textos de Saussure y de Naville aquí citados, resulta claro que la semiología es una ciencia psicológica, pues en todo caso atañe a las manifestaciones del espíritu humano, y más específicamente una ciencia sociológica, ya que se ocupa de tales manifestaciones en el transcurso del vivir social. La dimensión colectiva de los sistemas de signos es un punto tan importante que Saussure afirma claramente que la naturaleza social del signo es un factor intrínseco de todo sistema semiológico:

Solamente el sistema de signos convertidos en cosa de la colectividad merece el nombre de, es un sistema de signos: porque desde ese momento el conjunto de sus condiciones de vida es tan distinto de todo lo que puede constituirlo fuera de esto que el resto parece desprovisto de importancia.

7. No se olvide que Saussure (2002: 237) hasta llegó a emplear el término "signología" para hablar de la ciencia de los signos. Existe, por ende, un Saussure "alternativo" que propone una disciplina científica llamada "signología", cuyo principal objeto de estudio serían las "semiologías". 
$[\ldots]$

Llegados a este punto, vemos definirse, precisarse mejor el horizonte de la semiología, porque negamos que todo lo que se asemeja al signo tenga una naturaleza basada en las condiciones individuales, o más exactamente, sólo reconocemos como semiológica la parte de los fenómenos que aparece como producto social de modo característico. [...]

Sea cual sea su naturaleza más particular, la lengua, como las otras clases de signos, es ante todo un sistema de valores, y esto pone el fenómeno en su lugar. En efecto, toda clase de valor, aunque recurra a elementos muy diversos, solamente tiene su base en el medio y la potencia sociales. Es la colectividad la que es creadora del valor, lo cual significa que este no existe antes y fuera de aquella, ni en sus elementos descompuestos ni en los individuos (Saussure, 2002: 253-254).

Ahora bien, si es verdad que la "colectividad es necesaria para establecer valores cuya única razón de ser está en el uso y en el consenso generales" (Saussure, 1945 [1916]: 193) y que, por ende, la semiología se ocupa de hechos intrínsecamente sociales, cabe preguntar cómo resulta posible concebir los signos y sus "dos caras", el significado (o "concepto") y el significante (o "imagen acústica", en el caso de la lengua), como entidades meramente psicológicas, es decir, en este caso, entidades de conciencia.

Según Foucault (1966), el nexo binario entre la "idea" de una "cosa representante" (el significante) y la "idea" de una "cosa representada" (el significado) ya se hallaba en la gramática de Port-Royal y constituyó un punto firme en toda la reflexión acerca de los procesos sígnicos a lo largo de los siglos XVII y XVIII, de modo que, concluye Foucault (1966: 83), era inevitable que "Saussure, al retomar el proyecto de una semiología general, diera del signo una definición que ha podido parecer 'psicologista' (nexo de un concepto y de una imagen): en realidad, él de esta manera redescubría la condición clásica para pensar la naturaleza binaria del signo".

Pero este "redescubrimiento" no respondía a una operación de gusto 
filológico, sino a unas nuevas y fuertes exigencias de sistematización disciplinaria. En primer lugar, la lingüística, a través de la semiología (o de la "signología"), encontraba su "lugar natural" en la jerarquía de las ciencias humanas, según un sistema de inclusiones lógicas sucesivas, desde lo más específico hacia lo más general: lingüística ${ }^{\circledR}$ semiología ${ }^{\circledR}$ sociología ${ }^{\circledR}$ psicología (social); y se independizaba, asimismo, de aquellas disciplinas que hasta entonces habían mantenido el monopolio de los estudios lingüísticos: la gramática, la filología y la gramática comparada. En segundo lugar, los objetivos y la metodología de la lingüística no sólo encontraban el respaldo de los objetivos y la metodología de una ciencia más general, la semiología, sino que, siendo la lingüística la ciencia semiológica más avanzada y la lengua el más importante de los sistemas de signos, también encontraban un nuevo y fértil terreno de aplicaciones, comparaciones y generalizaciones explicativas. En tercer lugar, como veremos mejor en nuestro siguiente recorrido, reducir explicativamente los fenómenos observados a un conjunto limitado de leyes psicológicas subyacentes, aunque generadas por las dinámicas sociales $-\mathrm{y}$ es aquí que se injerta la "recuperación" señalada por Foucault-, presentaba la no desdeñable ventaja de poder excluir de los objetivos inmediatos de la disciplina el de dar cuenta del "caos" de las realizaciones individuales $\mathrm{y}$ de las contingencias externas (y espero con esto haber matizado convenientemente lo que sostuve en Lampis, 2016: 108).

Pero veamos, finalmente, también aquellos pasajes del Curso en que se presenta la nueva disciplina:

I

La lengua es un sistema de signos que expresan ideas, y por eso comparable a la escritura, al alfabeto de los sordomudos, a los ritos simbólicos, a las formas de cortesía, a las señales militares, etc., etc. Sólo que es el más importante de todos esos sistemas.

Se puede, pues, concebir una ciencia que estudie la vida de los signos en el seno de la vida social. Tal ciencia sería parte de la psicología social, y por consiguiente de la psicología general. Nosotros la llamaremos semiología (del griego sēmeîon, 'signo'). Ella nos enseñará en qué consisten los signos y cuáles son las leyes que los gobiernan. Puesto 
que todavía no existe, no se puede decir qué es lo que ella será; pero tiene derecho a la existencia, y su lugar está determinado de antemano. La lingüística no es más que una parte de esta ciencia general. Las leyes que la semiología descubra serán aplicables a la lingüística, y así es como la lingüística se encontrará ligada a un dominio bien definido en el conjunto de los hechos humanos.

Al psicólogo toca determinar el puesto exacto de la semiología; tarea del lingüista es definir qué es lo que hace de la lengua un sistema especial en el conjunto de los hechos semiológicos. Más adelante volveremos sobre la cuestión; aquí sólo nos fijamos en esto: si por vez primera hemos podido asignar a la lingüística un puesto entre las ciencias es por haberla incluido en la semiología.

¿Por qué la semiología no es reconocida como ciencia autónoma, ya que tiene como las demás su objeto propio? Es porque giramos dentro de un círculo vicioso: de un lado, nada más adecuado que la lengua para hacer comprender la naturaleza del problema semiológico; pero, para plantearlo convenientemente, se tendría que estudiar la lengua en sí misma; y el caso es que, hasta ahora, casi siempre se la ha encarado en función de otra cosa, desde otros puntos de vista.

\section{$[\ldots]$}

Para nosotros, por el contrario, el problema lingüístico es primordialmente semiológico, y en este hecho importante cobran significación nuestros razonamientos. Si se quiere descubrir la verdadera naturaleza de la lengua, hay que empezar por considerarla en lo que tiene de común con todos los otros sistemas del mismo orden; factores lingüísticos que a primera vista aparecen como muy importantes (por ejemplo, el juego del aparato fonador) no se deben considerar más que de segundo orden si no sirven más que para distinguir a la lengua de los otros sistemas. Con eso no solamente se esclarecerá el problema lingüístico, sino que, al considerar los ritos, las costumbres, etc., como signos, estos hechos aparecerán a otra luz, y se sentirá la 
necesidad de agruparlos en la semiología y de explicarlos por las leyes de esta ciencia (Saussure, 1945 [1916]: 60$62)$.

II

cuando la semiología esté organizada se tendrá que averiguar si los modos de expresión que se basan en signos enteramente naturales - como la pantomima-le pertenecen de derecho. Suponiendo que la semiología los acoja, su principal objetivo no por eso dejará de ser el conjunto de sistemas fundados en lo arbitrario del signo. En efecto, todo medio de expresión recibido de una sociedad se apoya en principio en un hábito colectivo o, lo que viene a ser lo mismo, en la convención. [...] Se puede, pues, decir que los signos enteramente arbitrarios son los que mejor realizan el ideal del procedimiento semiológico; por eso la lengua, el más complejo y el más extendido de los sistemas de expresión, es también el más característico de todos; en este sentido la lingüistica puede erigirse en el modelo general de toda semiología, aunque la lengua no sea más que un sistema particular (Saussure, 1945 [1916]: 130-131).

III

La continuidad del signo en el tiempo, unida a la alteración en el tiempo, es un principio de semiología general; y su confirmación se encuentra en los sistemas de escritura, en el lenguaje de los sordomudos, etc. (Saussure, 1945 [1916]: 143).

IV

hace falta una masa parlante para que haya una lengua. Contra toda apariencia, en momento alguno existe la lengua fuera del hecho social, porque es un fenómeno semiológico. Su naturaleza social es uno de sus caracteres internos (Saussure, 1945 [1916]: 144). 


\section{$\mathrm{V}$}

el sistema nunca es más que momentáneo: varía de posición a posición. Verdad que los valores dependen también, y sobre todo, de una convención inmutable, la regla de juego, que existe antes de iniciarse la partida y persiste tras cada jugada. Esta regla admitida una vez para siempre existe también en la lengua: son los principios constantes de la semiología (Saussure, 1945 [1916]: 159).

VI

en los sistemas semiológicos, como la lengua, donde los elementos se mantienen reciprocamente en equilibrio según reglas determinadas, la noción de identidad se confunde con la de valor y recíprocamente (Saussure, 1945 [1916]: 189).

\section{VII}

En la lengua, como en todo sistema semiológico, lo que distingue a un signo es todo lo que lo constituye. La diferencia es lo que hace la característica, como hace el valor y la unidad (Saussure, 1945 [1916]: 205).

Así pues, según Saussure, la semiología, a pesar de que todavía no exista ni podamos saber cómo será, tiene un lugar "determinado de antemano" en el sistema de las ciencias ('i'). Que esto sea así resulta evidente porque los seres humanos, en el transcurso de nuestros innumerables tratos sociales, sin duda empleamos y reconocemos signos y porque el uso de estos signos está seguramente reglamentado y responde, por ende, a leyes generales que pueden ser estudiadas por una disciplina específica.

La lingüística, de tal modo, encuentra un cómodo alojamiento en el edificio de las ciencias sociales $\mathrm{y}$, en última instancia, psicológicas. No se nos debe escapar aquí, sin embargo, una interesante petitio principii. La lingüística, sostiene Saussure, es sólo una rama especializada de la semiología, disciplina que todavía no existe y cuyo exponente más prestigioso sería... la propia lingüística. Así, todos los principios semiológicos generales enunciados por Saussure son principios referidos a la lengua para los que se supone, sin más, una generalización semiológica: 
- el signo es arbitrario ('ii') -es decir, es inmotivada la unión, el vínculo entre el significante y el significado-, siendo su único fundamento un "hábito colectivo" (de aquí, según De Mauro, la irreductible dimensión histórica de la lengua); incluso ahí donde se puede hablar de algún tipo de motivación (como en la pantomima, las palabras onomatopéyicas y las onomatopeyas), esta sólo sería parcial y conduciría, en cualquier caso, a fenómenos límites en el dominio semiológico;

- el signo tiene continuidad en el tiempo, aun cuando se altera ('iii'); es otro efecto de la arbitrariedad y de la "regencia" social del signo: el reconocimiento colectivo y continuo del signo es lo único que puede darle valor semiológico ('iv');

- el sistema de los signos es "momentáneo", esto es, puede ser aprendido, configuración tras configuración, sólo como una totalidad sincrónica de elementos co-presentes e inter-determinados (' $v$ ');

- el valor de un signo -su posición relativa a los signos con el que se combina sintagmáticamente y con los que entra en relaciones asociativas (por sinonimia, antonimia, analogía, etc.)- constituye su identidad, su reconocibilidad en tanto que signo ('vi'); es, en otros términos, la diferencia de un signo con respecto a los demás signos del sistema lo que lo individúa como signo y determina su función ('vii').

Todos estos principios, aplicables al sistema de la lengua, pueden ser extendidos también a los demás sistemas de signos; se da por sentada, pues, la legitimidad de tal extensión, aunque es la aplicabilidad semiológica general de los principios lo que (también) debería legitimarlos como asertos científicos.

Cabe recordar que la lingüística, así como vaticinó Saussure y por los mismos motivos que él adujo, se impuso con frecuencia -y aún hoy en día sigue imponiéndose- como modelo para las demás disciplinas semióticas. Como también cabe recordar que la "crisis" del modelo semiolingüístico saussureano se debió, precisamente, a su inaplicabilidad a muchas manifestaciones semiósicas de tipo continuo y analógico -incluidas muchas manifestaciones discursivas- $\mathrm{y}$ a la exigencia de proponer modelos explicativos que pudieran dar cuenta de la diversidad y la heterogeneidad internas de los diferentes sistemas semióticos investigados. 


\section{REFERENCIAS BIBLIOGRÁFICAS}

BENVENISTE, E. (1975). "Semiologia della lingua". En Semiotica in nuce. Volume II. Teoría del discorso, P. Fabbri y G. Marrone (eds.), 86-98. Roma: Meltemi, 2001.

BERTRAND, D. (2000). Basi di semiotica letteraria. Traducción de A. Perri. Roma: Meltemi, 2002.

COQUET, J.-C. (2008) Le istanze enuncianti. Semiotica e fenomenologia. Traducción de E. Nicolini. Roma: Bruno Mondadori.

DE MAURO, T. (1967a). Introduzione. En Saussure, 1967 [1922]: V-XXIII.

(1967b). Notizie biografiche e critiche su F. de Saussure / Note. En Saussure, 1967 [1922]: 285-456.

DUCROT, O. y TODOROV, T. (1972). Diccionario enciclopédico de las ciencias del lenguaje. Traducción de E. Pezzoni. México: Siglo XXI, 2009.

FABBRI, P. y MARRONE, G. (eds.) (2000). Semiotica in nuce. Volume I. I fondamenti e l'epistemologia strutturale. Roma: Meltemi.

FOUCAULT, M. (1966). Le parole e le cose. Un'archeologia delle scienze umane. Traducción de E. Panaitescu. Milano: Rizzoli, 1998.

GONZÁlEZ DE ÁVILA, M. (2002). Semiótica crítica y crítica de la cultura. Barcelona: Anthropos.

HJELMSLEV, L. (1961). I fondamenti della teoria del linguaggio. Introducción y traducción de G. C. Lepschy. Torino: Einaudi, 1968.

LAMPIS, M. (2016). Tratado de semiótica caótica. Sevilla: Alfar.

NAVILLE, A. (1901). Nouvelle classification des sciences. Étude philosophique. París: Félix Alcan.

SAUSSURE, F. de (1916). Cours de linguistique générale. Edición crítica preparada por T. De Mauro. París: Payot, 2005.

(1945 [1916]). Curso de lingüistica general. Traducción, prólogo y notas de A. Alonso. Buenos Aires: Losada, 1973.

(1967 [1922]). Corso di linguistica generale. Introducción, traducción y comento de T. De Mauro. Roma-Bari: Laterza, 2005.

(2002). Escritos de lingüistica general. Edición de S. Bouquet y R. Engler. Traducción de C. U. Lorda Mar. Barcelona: Gedisa, 2004.

ŠTÚR, M. (2013). "Three Basic Language and Semiotic Dichotomies after One Century of Development and the Complexity of Semiotic 
System". XLingue Journal, 6.4, 3-17.

Recibido el 6 de marzo de 2017.

Aceptado el 16 de abril de 2017. 